\title{
TÉCNICA MFR PARA PERSONALIZAR EL PLANO DE FOX Y DETERMINAR EL PLANO OCLUSAL.
}

\section{MFR TECHNIQUE TO CUSTOMIZE THE FOX PLANE AND DETERMINE THE OCCLUSAL PLANE.}

\author{
Milton Flor Rodríguez ${ }^{1}$ \\ ${ }^{1}$ Especialista en Rehabilitación Oral. Docente de la Universidad Nacional Jorge Basadre Grohmann, Tacna-Perú.
}

\begin{abstract}
Resumen
El plano oclusal es una superficie arquitectónica que al igual que el plano de una casa no se hace evidente hasta que sea construido, siendo esencial para lograr su total edificación. En algunos pacientes es muy difícil poder determinar un plano oclusal ideal por tal motivo el presente artículo tiene el objetivo de proponer una técnica para personalizar el Plano de Fox y determinar el plano oclusal en pacientes edéntulos parciales. Para lograr esta propuesta se modificó el plano de Fox con la finalidad de determinar el plano oclusal de pacientes edéntulos parciales, esta técnica se desarrolló en la cátedra de Oclusión de la Universidad Nacional Jorge Basadre Grohmann de la ciudad de Tacna-Perú. Desde la perspectiva del autor la aplicación de la técnica MFR de Modificación del plano de Fox para la Rehabilitación Oral de pacientes Edéntulos Parciales, es una alternativa clínica que nos facilita el diagnóstico y el plan de tratamiento.
\end{abstract}

Palabras clave: Relación Céntrica, Plano Oclusal, Dimensión Vertical.

\begin{abstract}
The occlusal plane is an architectural surface that, like the plan of a house, does not become evident until it is built, being essential to achieve its total construction. In some patients it is very difficult to determine an ideal occlusal plane, for this reason the present article aims to propose a technique to personalize the Fox Plane and determine the occlusal plane in partial edentulous patients. To achieve this proposal, the Fox plane was modified in order to determine the occlusal plane of partial edentulous patients. This technique was developed in the Occlusion chair of the Jorge Basadre Grohmann National University in the city of Tacna-Peru. From the author's perspective, the application of the Fox plane Modification MFR technique for the Oral Rehabilitation of Partial Edentulous patients is a clinical alternative that facilitates the diagnosis and the treatment plan.
\end{abstract}

Key words: Centric Relationship, Occlusal Plane, Vertical Dimension.

\section{Introducción}

Actualmente, The Glossary of Prosthodontic Terms (2005) define el Plano Oclusal clínico como "el plano promedio establecido por las superficies incisales y oclusales de los dientes. Generalmente no es un plano, pero representa el plano medio de la curvatura de esas superficies". Se deberá tener presente el término "plano" que en biología se refiere a una superficie definida por tres puntos en el espacio, como mínimo, lo que no significa que, los tres puntos estén en línea recta. No obstante, se considera viable el uso del término "plano" en las referencias para la construcción de las prótesis totales artificiales. ${ }^{1}$ El plano oclusal es una superficie arquitectónica que al igual que el plano de una casa no se hace evidente hasta que sea construido, siendo esencial para lograr su total edificación.

El edentulismo es la pérdida de los dientes permanentes en una persona adulta. Tiene distintos grados de afección que pueden ser: parcial y total, pudiendo padecerlo hombres y mujeres, sin límite de edad, etnia o color de la piel, ${ }^{2}$ no es exclusivo del adulto mayor, como es común pensar; hay diversas causas que hacen que la franja de edad afectada sea muy amplia, factores a propiciarlo pueden ser: deficiente higiene bucal, traumatismos, enfermedades de etiologías múltiples e incluso el uso, abuso o dependencia de medicamentos o drogas, sin predilección en ambos sexos. ${ }^{3}$ Gran parte de ellos requieren se determine el plano oclusal que sirva como base de su rehabilitación. 
Para poder llegar a un acertado diagnóstico de patología oclusal así como para planificar el tratamiento de nuestros pacientes, es necesario, ordenar los procedimientos que nos lleven a conseguir nuestros objetivos, considerando como punto de inicio y de término una correcta posición condilar en la zona articular de su respectiva cavidad glenoidea; ${ }^{4}$ seguido de que debemos establecer un plano oclusal ideal y personalizado, lo que nos permitirá cumplir con la distribución equilibrada de las fuerzas masticatorias que se imprimen sobre el sistema dentario, compartiendo éstas por igual tanto entre las articulaciones alveolo dentarias como en las articulaciones temporomandibulares, obteniendo una Oclusión Mutuamente Compartida, a su vez este plano oclusal ideal nos permite una correcta alineación dentaria en su posición de zona neutra, Alonso ${ }^{5}$ denomina estas características como alineación tridimensional; finalizando con una Dimensión Vertical que será adecuada y proporcional, respetando parámetros fisiológicos y estéticos.

Por lo expuesto, la técnica MFR propone conseguir tres objetivos fundamentales:

- Establecer una correcta posición condilar.

- Personalizar el Plano de Fox modificándolo para obtener un Plano Oclusal ideal.

- Determinar una Dimensión Vertical adecuada y proporcional.

\section{Descripción de la Técnica}

La Técnica MFR para determinar el plano oclusal, se basa en conseguir el paralelismo del plano oclusal con el plano de Camper, y en el sector anterior el paralelismo con el plano bipupilar, en pacientes que presenten su alteración. ${ }^{6}$
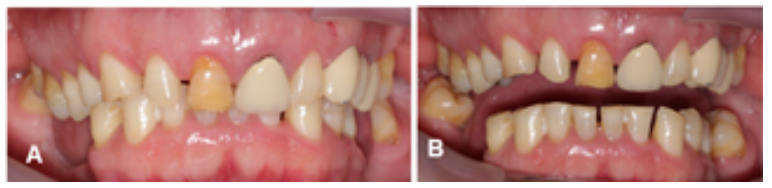

Fig. 1. Fotografias intraorales paciente parcialmente edéntulo

Es importante para la técnica el análisis de la sonrisa del paciente, para determinar cuanto de encía muestra al sonreír, evaluando el biotipo gingival (con estos datos podemos planificar la terminación cervical del preparo de la pieza dental así como el material para la restauración); obtendremos el porcentaje que ocupa el incisivo central superior del espacio que existe entre el borde inferior

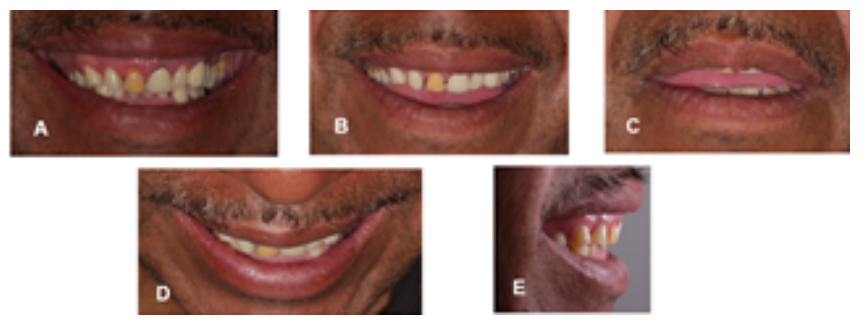

Fig. 2. Fotografías para el análisis de la posición de las piezas antero superiores.

del bermellón del labio superior al borde superior del bermellón del labio inferior, considerando que el incisivo central superior debiera ocupar entre el $50 \%$ y el $80 \%$ de este espacio. ${ }^{7}$ Ernest Mallat ${ }^{8}$ explica que la pronunciación de la letra $\mathrm{M}$ como parte de la técnica fonética en el establecimiento de la dimensión vertical, a su vez es útil para establecer la longitud y posición del del incisivo central superior, evaluando la posibilidad de su modificación en base a parámetros estéticos y a la proporción aurea. ${ }^{9}$

El registro de la relación céntrica es un paso vital en el diagnóstico, plan de tratamiento y en el tratamiento propiamente dicho, es el punto de inicio y el punto final de todo proceso en rehabilitación. Resulta ser el primer paso decidir la posición condilar de trabajo. ${ }^{10}$ Existen varios métodos que nos permiten obtener este registro, todos ellos buscan la desprogramación neuromuscular. Al utilizar como desprogramador el Jig de Lucia se debe lograr que solo un incisivo central inferior contacte con la superficie del Jig, luego verificaremos la desprogramación quitando el jig de Lucia y al cierre el paciente debe ubicar el lado donde percibe el primer contacto (Retrusivo de Contacto), indicamos al paciente que cierre completamente haciendo contactar todas sus piezas dentarias, observaremos un deslizamiento que va del retrusivo de contacto a máxima intercuspidación, sería el deslizamiento en céntrica; ${ }^{5,11,12}$ al obtener un punto repetitivo de contacto al cierre en el Jig se aplica adhesivo con un brush, procedemos a colocar una porción de resina compuesta, indicamos al paciente que eleve el maxilar inferior hasta que el incisivo inferior contacte el jig de Lucia en la marca dejada por el papel de articular, se procede a fotocurar, y registramos esta posición con el material para registro.

Para la Técnica MFR se debe tener dos juegos de modelos, el primer juego de modelos se articula en la posición de relación céntrica y mediante el arco facial del articulador, realizar la transferencia de la posición que tiene el maxilar superior en el macizo cráneo facial.

El segundo juego de modelos lo utilizamos para modificar el plano de Fox buscando el paralelismo con el 

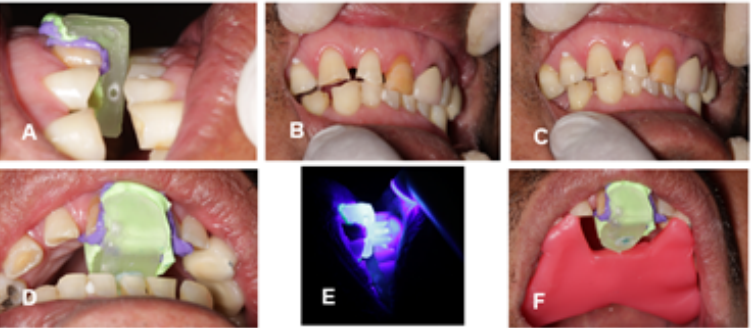

Fig. 3. Registro de la posición en Relación Céntrica.
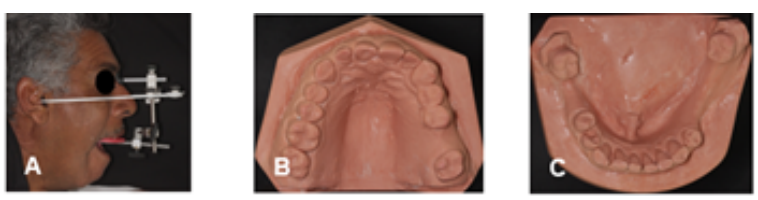

Fig. 4. Transferencia de la posición del maxilar superior al articulador mediante el arco facial.

plano bipupilar y el plano de Camper.
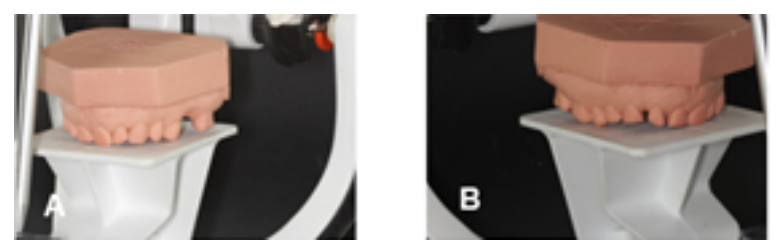

Fig. 5. Apoyamos los modelos en la mesa de Camper del articulador para determinar las posibles piezas dentarias que evitan el paralelismo de los planos.

Personalizar el Plano de Fox modificándolo para obtener un Plano Oclusal ideal, luego de la evaluación clínica del plano oclusal que presenta el paciente utilizando el plano de Fox, obtenemos el lugar donde se debe modificar para conseguir el paralelismo del plano oclusal con el plano de Camper. 6,13
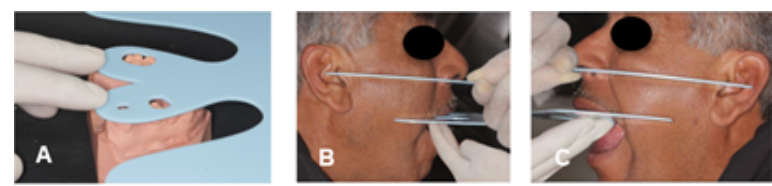

Fig. 6. Modificación del plano de Fox y búsqueda del parelelismo con el plano de Camper.

Terminada esta etapa clínica, hemos conseguido un plano oclusal ideal, resultando fácil identificar las piezas dentarias que no se encuentran en el plano ya sea por extrusión o por intrusión, dando parámetros para la alineación tridimensional. Es hora de verificar que los datos clínicos obtenidos son los mismos que tendremos en los modelos de diagnóstico en el articulador semiajustable.
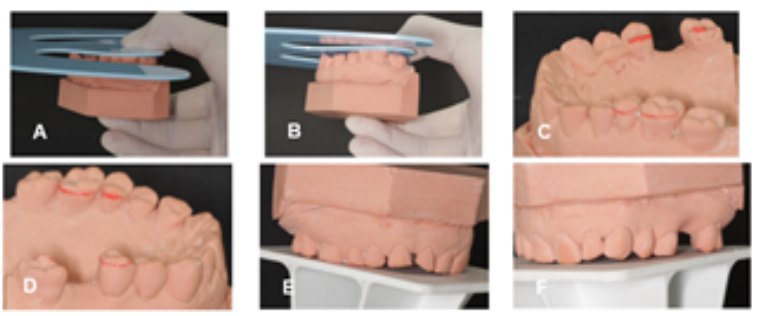

Fig. 7. Verificar los datos clínicos en los modelos de estudio.

Una vez nivelado el plano oclusal del modelo, se procede a realizar el montaje en la mesa de Camper del articulador, respetando las líneas de referencia de la mesa o ubicando en ella una plantilla de proporciones áureas. El modelo inferior se articula con el superior usando el registro de la posición céntrica del primer juego de modelos.

Determinar la dimensión vertical fisiológica y proporcional en la que planificaremos el tratamiento. Existen diferentes técnicas que nos permiten determinar la dimensión vertical, ninguna es determinante, pero sí referenciales, en el presente caso clínico y en la mayoría de los resueltos, usamos la combinación de las técnicas métricas, fonéticas, deglutorias, obteniendo buenos resultados. $8,10,12,14$

Una vez determinada la dimensión vertical, fisiológica y proporcional, en la que realizaremos la planificación del tratamiento, se procede a realizar el encerado diagnóstico.

La ventaja de utilizar la técnica MFR para conseguir el paralelismo mediante la Modificación del plano de Fox radica en la obtención directa de datos clínicos que serán analizados en los modelos de estudio, permitiendo el diagnóstico y la planificación del tratamiento, de manera sencilla, clínica y de bajo costo.

Considerando que, a diferencia de técnicas convencionales, en la técnica MFR iniciamos el encerado en el maxilar superior estableciendo la posición correcta, así como la longitud ideal en ancho y alto del incisivo central superior, basados en la proporción áurea. La posición del incisivo central es de vital importancia, ya que es el inicio de un plano oclusal ideal y el punto de partida de una correcta dimensión vertical. ${ }^{8}$ Recordemos que las piezas anteriores forman la guía anterior responsable de la desoclusión. ${ }^{5,15} \mathrm{La}$ alineación tridimensional o la posición de zona neutra del sector posterior siguen los parámetros del enfilado dentario de la prótesis completa. 

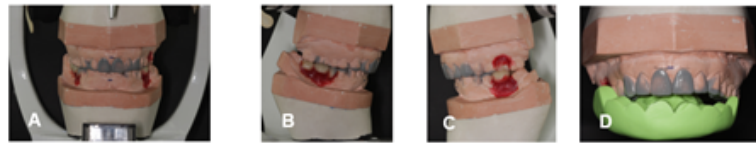

Fig. 8. Encerado diagnóstico y llave de silicona para Mock up anterior.
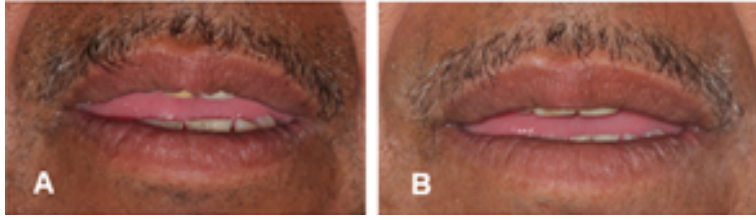

Fig. 9. B) Fotografia pronunciando la letra $M$ sin mock up. C) Fotografía pronunciando la letra M con Mock up.
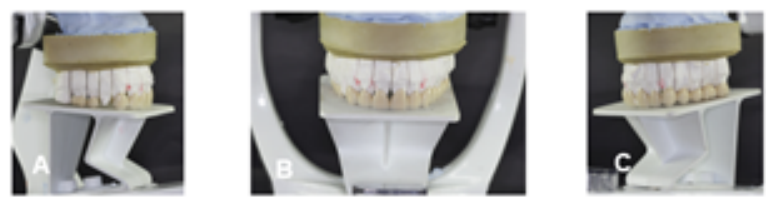

Fig. 10. Se verifica la correcta alineación tridimensional con respecto a la mesa de camper, respetando la formación de curvas, Spee y Wilson, así como la perpendicularidad de los centrales con el plano horizontal. A) lateral derecha, pieza 14 será restaurada con overlay. B) Vista frontal. C) Vista lateral izquierda.
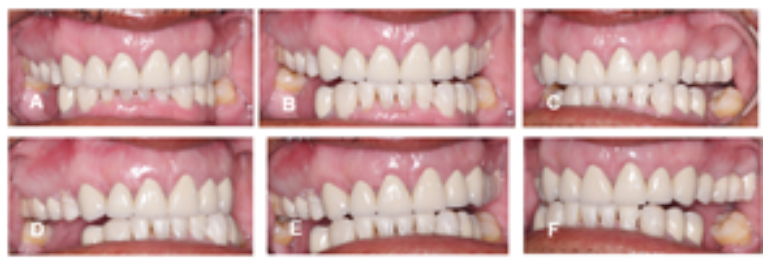

Fig. 11. Oclusión y Desoclusión. A) En oclusión vista frontal. B) Movimiento protrusivo, observese el fenómeno de Christensen, espacios uniformes en el sector posterior. C) Movimiento de lateralidad izquierda, lado de trabajo. D) Lateralidad izquierda, lado de balance. E) Lateralidad derecha, lado de trabajo. F) Lateralidad izquierda, lado de balance.
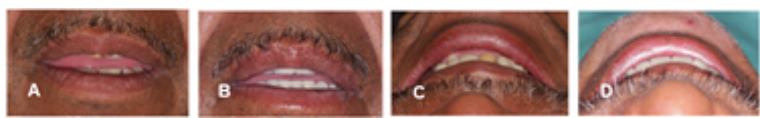

Fig. 12. Antes y después. A y B) Pronunciando la letra M. C y D) Fotografía a las 12.

Conflicto de intereses: Los autores del presente estudio manifiestan que no existe ningún conflicto de intereses en relación al tema de estudio.

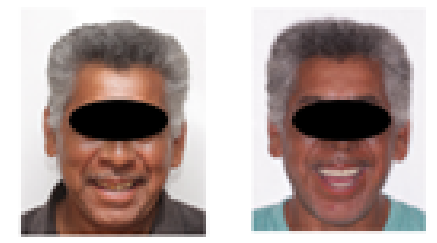

Fig. 13. Fotografía extraoral. A) Antes. B) Después.

\section{Referencias}

1 Schunke S. Reflexiones sobre el complejo temático funcional a partir de diversas reconstrucciones $2^{\circ}$ parte. Quintessenz Zahntech 2008; 34(6):664-72.

2 Organización Mundial de la Salud [Internet]. Organización Mundial de la Salud; c2012 [citado 2 ene 2018]. Salud Bucodental; [aprox.4 p.]. Disponible en: http://www.who.int/mediacentre/factsheets/fs318/es/

3 Alvítez D. Dimensión vertical oclusal. Artículo de revisión. Odontol Sanmarquina. 2016; 19(1): 56-60.

4 Loza D. Oclusión. 1ra Edición. Lima. Savia Editorial SRL. 2015.

5 Alonso, A. A. Oclusión y Diagnóstico en rehabilitación Oral. Editorial Médica Panamericana. 2004.

6 Sacerdote G, Wilson MG. Una evaluación de los puntos de referencia para la orientación estética del plano oclusal. J Prosthodont. Abril de 2017; 26 (3): 216-223. doi: 10.1111 / jopr.12524. Publicación electrónica del 29 de julio de 2016. PMID: 27472047.

7 Fradeani M. Rehabilitación estética en prostodoncia fija: análisis estético. 1ra Edición. Quintessence Publishing Co, Ltd. 2015.

8 Mallat E. Las claves de la prótesis fija en cerámica. 1ra Edición. Valencia. Lisermed Editorial SL. 2018.

9 Carvalho P. Carillas: lentes de contacto y fragmentos cerámicos. 1ra Edición. Florianapolis. Editorial Ponto Ltda. 2015.

10 Dawson P. Oclusión Funcional: diseño de la sonrisa a partir de la ATM. 1ra Edición. St Louis, Missouri. Actualidades Médico Odontológicas Latinoamericana, CA. 2009.

11 Jiménez-Silva A, Tobar-Reyes J, Vivanco-Coke S, PasténCastro E, Palomino-Montenegro H. Discrepancia relación céntrica-posición intercuspal y su relación con los trastornos temporomandibulares. Una revisión sistemática. Acta Odontol Scand. Octubre de 2017; 75 (7): 463-474. doi: 10.1080 / 00016357.2017.1340667. Publicación electrónica del 22 de junio de 2017 PMID: 28641068.

12 Okeson, J. P. Tratamiento de Oclusión y Afecciones Témporomandibulares. Editorial Elsevier España. 2020.

13 García J. Enfilado dentario bases para la estética y la estática en prótesis totales. 1ra Edición. Colombia. Actualidades Médico Odontológicas Latinoamericana, CA. 2009.

14 Calamita M, Coachman C, Sesma N, Kois J. Dimensión vertical oclusal: decisiones de planificación del tratamiento 
y consideraciones de manejo. Int J Esthet Dent. 2019; 14 (2): 166-181. PMID: 31061997.

15 Manns, Arturo E. Sistema estomatognático. Fundamentos clínicos de fisiología y patología funcional. Editorial Amolca. 2013.

Recibido: 11 de diciembre de 2020

Aceptado: 12 de febrero de 2021 
